\title{
Research on data transmission method of new media marketing analysis based on Web4.0
}

\author{
WU Qi-xiang ${ }^{1}, \mathrm{HE}$ Lin $^{2}$, ZHOU Xian-chun ${ }^{3}$ \\ \{ainiyiwantian255@tom.com ${ }^{1}$, hupeng12123@sohu.com²,wangyang19111@163.com ${ }^{3}$ \} \\ (1. College of Information Engineering,Fuyang normal university, fuyang 236041, China; \\ 2.College of Information Engineering,Fuyang normal university, fuyang 236041, China; \\ 3.School of Information and Intelligence Engineering,Sanya University, Hainan Analysis and \\ Application Engineering Research Center of Software Gene Virus,Sanya 572022, China)
}

\begin{abstract}
The energy consumption of existing data transmission methods is too high, so a new media marketing analysis data transmission method based on Web4.0 is designed. By designing the structure diagram of transmission method, the reliable data transmission in maintaining system and at the same time as far as possible to reduce the node energy consumption and data transmission of Steiner tree model is set up, cut it off and distributed data do not need the link, reduce the amount of calculation, the design parameters of transmission channel, the research of inductive coupler circuit inductance and the resistance value, on the basis of reducing one hop communication distance, multipath allocation process design, complete the multipath optimization allocation mechanism. So far, the research on the data transmission method of new media marketing analysis based on Web4.0 has been completed. The experimental results show that the designed transmission method can save $176 \mathrm{KWH}$ compared with the existing data transmission method.
\end{abstract}

Key words: New media marketing; Data transmission; Transmission channel parameters;

\section{Introduction}

With the development of information technology and Internet technology, web4.0 has gradually integrated into our lives. Under the premise of identity recognition, it integrates all the digital resources and information resources that can be integrated to provide the best information services for users. In this context, many products are also faced with the environment changed by new production technology and new marketing methods ${ }^{[1-2]}$. For consumers, the new media marketing under Web4.0 allows everyone to experience products from all over the world without leaving home, shortening the time, space, and home delivery experience, which is wrapping up more and more people Becoming a new force in new consumption patterns has also allowed the online sales scale of various products to continue to break through.

In order to increase product sales and understand the status of online marketing, 
marketing analysis data needs to be transmitted. In the existing transmission method, the energy consumption of the transmission node is too high, so a new media marketing analysis data based on Web 4.0 is designed. Research on transmission methods. By designing the structure block diagram of the transmission method, the Steiner tree model of data transmission is established to reduce and balance the energy consumption of nodes as much as possible while ensuring the reliable transmission of the system data. In the process of data distribution, unnecessary links are cut off, the calculation amount is reduced, the parameters of the transmission channel are designed, the circuit of the inductive coupler is studied, and the inductance value and impedance value are obtained On the basis of reducing the single hop communication distance, the multi-path allocation process is designed and the multi-path allocation mechanism is optimized.

\section{Research on Data Transmission Method of New Media Marketing Analysis Based on Web4.0}

With the increasing application of Internet Web 4.0, the requirements for data transmission reliability have become higher and higher. Due to the limited energy resources of the nodes in the Internet, when the energy of the nodes is reduced, the communication capacity of the nodes is significantly reduced, which further reduces the reliability of the forwarded packets. Once the nodes do not work properly, the network topology will change, which will affect the data transmission efficiency and overall reliability of the sensor network. Therefore, it is necessary to reduce and balance node energy consumption as much as possible while improving reliability ${ }^{[3-4]}$. Because the energy-saving of nodes and the reliability of the system depend on the network structure to a great extent, only from the perspective of network structure can we design an efficient and reliable data transmission strategy, and establish a network structure that takes into account the energy-saving of nodes and the reliability of the system. The block diagram of the design transmission method structure is shown below: 


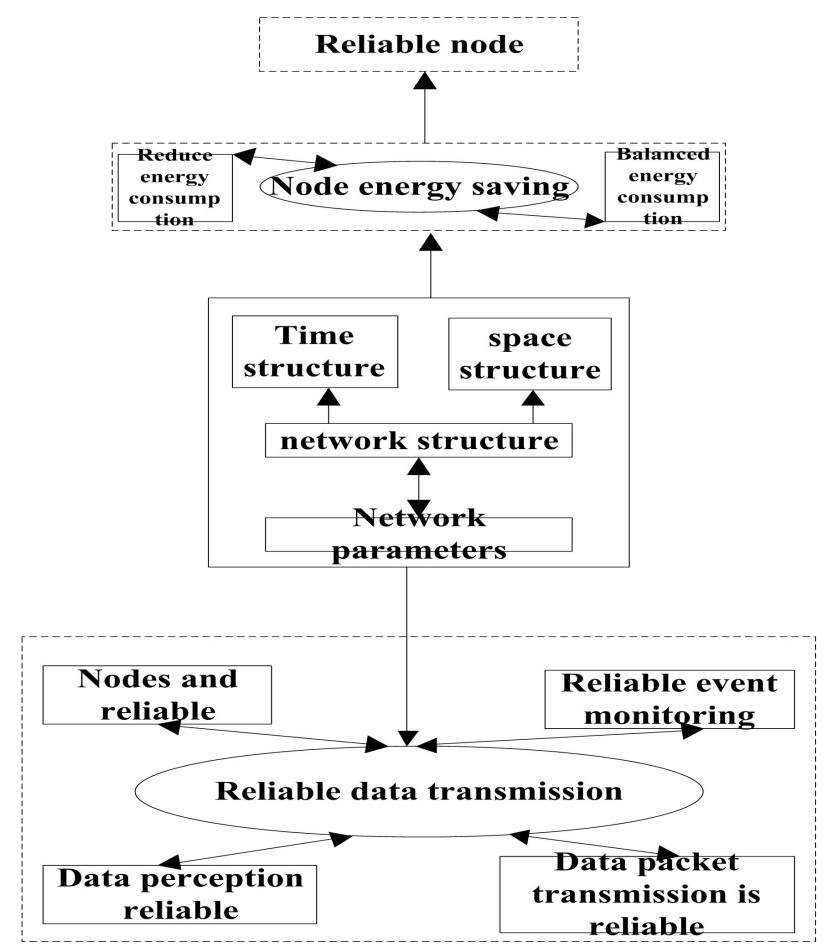

Fig1 Transmission method structure block diagram

The network structure is mainly composed of time structure and space structure. Its model is usually expressed by network parameters. Different network structure determines the properties of each network parameter, and the different values of each network parameter will make the network structure save energy on the nodes with different performance, and make the reliable transmission of system data different. Therefore, the key to the construction of the network structure is how to consider from the time and space structure of the network to reduce and balance the energy consumption of the node as much as possible while maintaining the reliable transmission of system data. The basis for reliable transmission applications.

\subsection{Building a data transfer model}

Modern data center networks provide multiple discontinuous Steiner trees for reliable group data transmission. Using these disjoint trees to perform data transmission in parallel can effectively improve the transmission rate of reliable group data transmission ${ }^{5-6]}$. Therefore, multiple data disjoint Steiner tree models in the data center are used to complete the data distribution. The Steiner tree structure is shown below: 


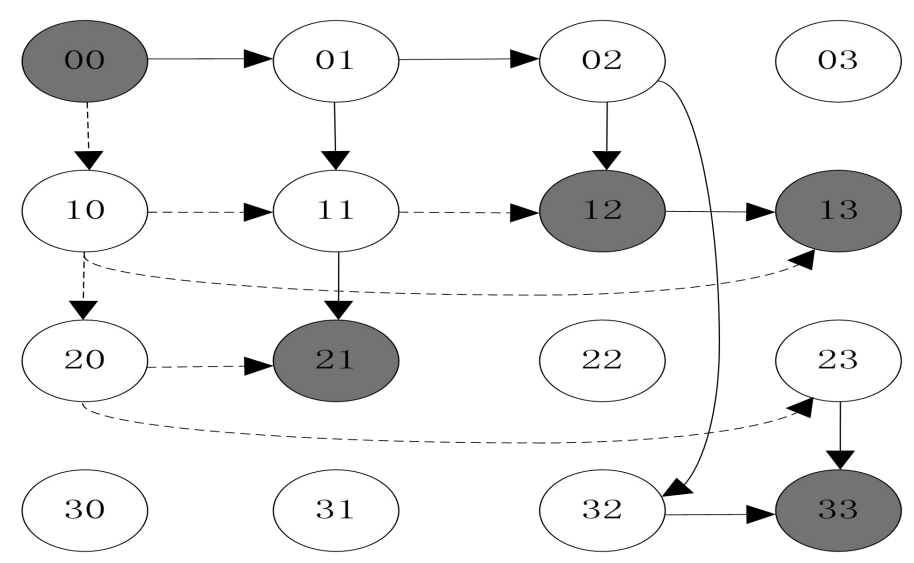

Fig2 Steiner tree structure

As can be seen from the above figure, the Steiner tree is a tree with the data source as the root node and all receiving nodes. In the figure above, the data transmission method based on the packet buffer is also shown. The solid line is the Steiner tree A, and the dashed line is the Steiner tree B. Assume that 00 is the source node, 12, 13, 21, and 33 are the data receiving nodes. Compared with the general creation of multiple spanning trees, the structural model has the advantage that the time complexity is much smaller than the general model, and the calculation can be completed in a short time. Secondly, the spanning tree height calculated by this model is higher than that of the general model. The model is small and similar to the network radius. After computing multiple edge disjoint spanning trees for the data center network, we calculated multiple Steiner trees by pruning, that is, cutting off the links that are not needed for data distribution. The process is shown in the following figure 3

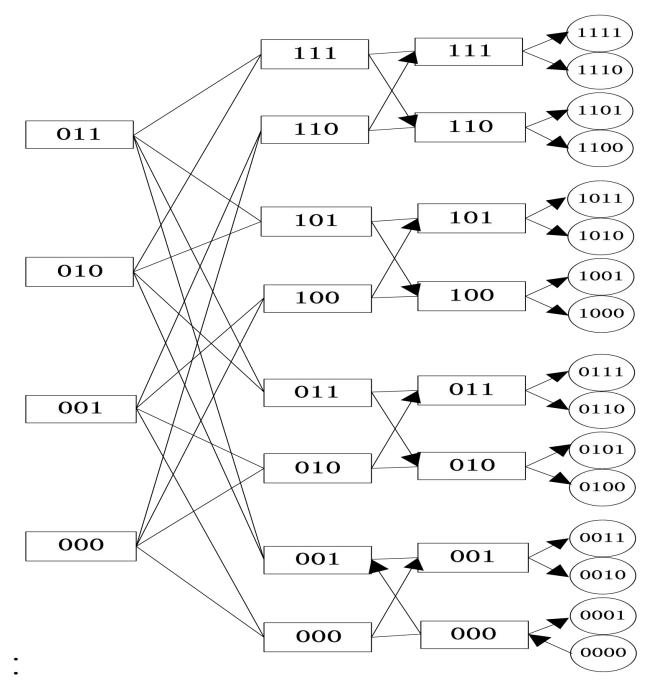


Fig3 Link pruning process

After computing multiple edge disjoint spanning trees for the data center network, we calculated multiple trees by pruning, that is, pruning the links that are not needed for data distribution. In the above figure, when 0001 is not a receiving node, the link from switch 000 to node 0001 can be reduced. In order to prun the spanning tree, we only need to calculate the set of paths from each node to the source node in the spanning tree. This can reduce the amount of calculation and complete the establishment of the data transmission model.

\subsection{Designing the parameters of the transmission channel}

The data transmission channel is formed by multiple inductive couplers through wireless high-speed data transmission connections. The electromagnetic inductive coupler consists of a ferrite core and a coil. This article uses nickel-zinc ferrite to combine the two ferrites Its physical dimensions are shown below:

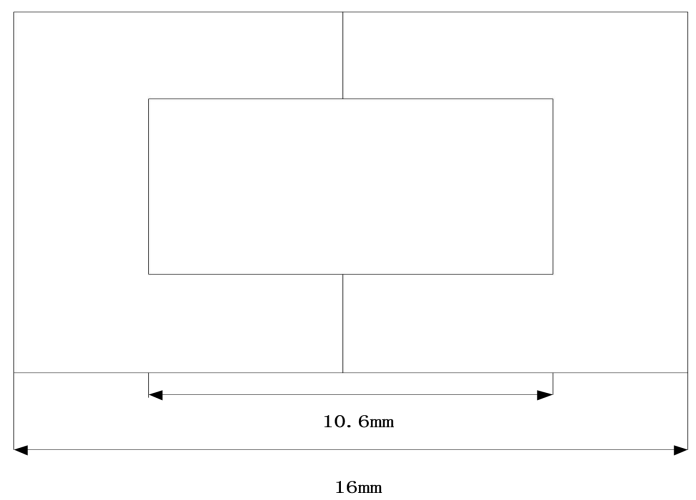

Fig4 Coupler size chart

In the coupler, the initial magnetic permeability is $\mu_{i}=200$, the specific resistance is $\rho=140 \Omega \mathrm{gm}$, and the Curie temperature is $T_{c}>130{ }^{\circ} \mathrm{C}$. Because the demodulation chip used in the previous stage is unstable, the carrier frequency should be selected at the low and medium frequencies, and the frequency of the signal at $270 \mathrm{kHz}$ Skin depth is:

$$
\Delta=\sqrt{\frac{2}{\mu_{i} \rho}}(1)
$$

The skin depth can be calculated, and it is determined that the skin diameter is doubled, and the number of coil turns of the coupler is 50 turns. The circuit of the inductive coupler is very similar to the high-frequency transformer. Based on the high-frequency transformer, the 
circuit of the inductive coupler is designed:

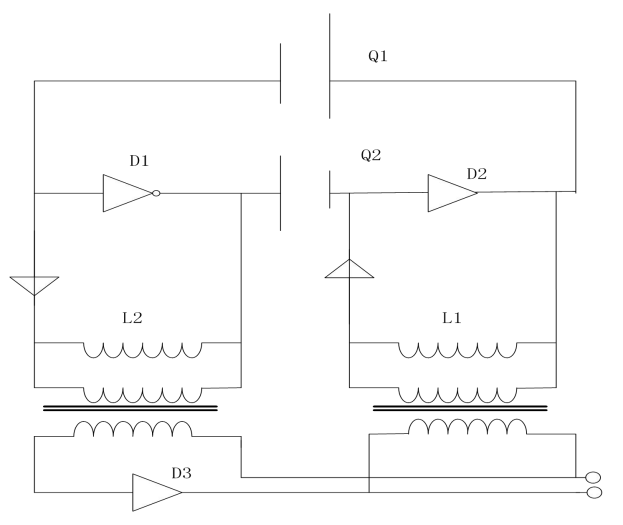

Fig5 Circuit design of inductive coupler

Because the inductive coupler is symmetrical in the primary and secondary, all parameters in the circuit model can be considered to be symmetrical. The amplifier $D_{1}$ in the circuit represents the DC loss of the inductive coupler coil. Because it has leakage inductance, the inductive coupler and the ideal transformer model differ in some characteristics ${ }^{[7-8]}$. Use an RLC automatic measuring instrument to measure one side of the inductive coupler at different frequencies $f$. It is equivalent to the inductance and the coil impedance in series. The inductance and impedance values are shown in the following table:

\begin{tabular}{ccc}
\multicolumn{2}{c}{ Tab1 Self-inductance measurement of inductive coupler coils at different frequencies } \\
\hline$f(\mathrm{kHz})$ & $L(\mathrm{mH})$ & $R(\Omega)$ \\
\hline 50 & 1.042 & 7.56 \\
100 & 1.073 & 11.8 \\
150 & 1.121 & 17.98 \\
200 & 1.172 & 25.33 \\
250 & 1.26 & 35.64 \\
300 & 1.34 & 54.14 \\
350 & 1.49 & 67.52 \\
400 & 1.55 & 71.32 \\
450 & 1.68 & 80.36 \\
\hline
\end{tabular}

Through the RLC automatic measuring instrument at the frequency of $450 \mathrm{kHz}$, the 
self-inductance deviation of the coupler was measured within $1.04-1.6 \mathrm{mH}$. Considering the frequency range of the data transmission channel of the experimental system, $L$ was selected as $1.3 . \mathrm{mH}$, and $R$ was selected as $50 \Omega$. This completes the design of the channel parameters.

\subsection{Optimize multi-path allocation mechanism}

In the practical application of data transmission, the energy consumption between different links will be different due to a series of effects. Therefore, it is necessary to optimize the multi-path allocation mechanism and add transmission consumption weights for each link, so that the network can better integrate with the objective environment in which it is located, thereby better improving the reliability of data transmission. Due to the influence of the topography, such as mountains, buildings, obstacles, and natural environments such as storms and lightning, the performance of wireless communications may change frequently, and communication interruptions frequently occur. These factors determine the value of the transmission consumption weight. . Ability to calculate the transmission consumption weight matrix:

$$
W=\left(w_{i j}\right)_{n \times n}
$$

among them:

$$
w_{i j}= \begin{cases}w_{i j}^{*} & e_{i j} \in E \\ 0 & e_{i j} \notin E\end{cases}
$$

In the above formula, $e_{i j}$ is the link between nodes $V_{i}$ and $V_{j}, w_{i j}^{*}$ is the transmission consumption weight of the link between nodes $V_{i}$ and $V_{j}$, and $0 \leq w_{i j}^{*} \leq 1 . E$ is the link set, $n$ is the number of middle nodes, and $2<n<4$. It is known that the energy consumption $P$ of the data transmission and the communication distance $d$ exist:

$$
P=k d_{i j}^{n}(4)
$$

In the above formula, $k$ is the energy parameter and $d_{i j}$ is the distance between nodes $V_{i}$ and $V_{j}$. It can be seen that as the communication distance increases, the energy 
consumption will increase sharply, and the single-hop communication distance should be minimized on the premise of satisfying the communication connectivity. Therefore, a multi-path allocation process can be designed:

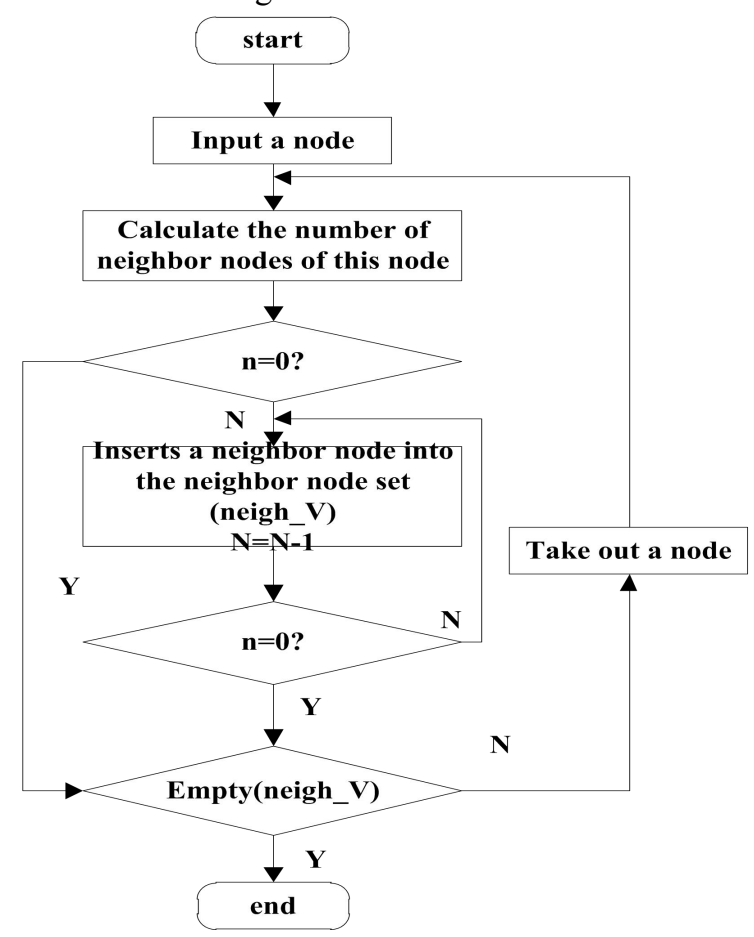

Fig6 Multi-path allocation process

The base station extracts the node information traversed by the data packet and the corresponding signal strength parameters, and converts all the path request data to the base station into a graph. The algorithm designed in this paper is used to generate the path from any node extracted from the node set to the base station. In order to obtain the multi-path routing from a node to the base station to the greatest extent, the idea of breadth-first algorithm is used in the path generation process $^{[9-10]}$. This completes the optimization of the multi-path allocation mechanism.

\section{Experiment}

In order to verify the effectiveness of the Web4.0-based new media marketing analysis data transmission method designed in this paper, it is necessary to perform a power consumption test to verify its energy saving effect.

\subsection{Experiment preparation}

In different branch lines of the same data transmission line, the transmission method designed in this paper and the existing transmission method are used to randomly select a 
continuous period (24 hours) as the test stage, and the energy consumption monitoring equipment gem-view is used to monitor the energy consumption of the two systems respectively. Firstly, the energy consumption of the system designed in this paper is monitored, and then the energy consumption of the traditional system is monitored. The equipment has the functions of basic energy consumption measurement and billing and report analysis, and provides online energy consumption detection (up to second level), energy consumption diagnosis and analysis, etc. its parameters are as follows:

Tab2 GEM-VIEW parameter table

\begin{tabular}{ccc}
\hline Features & project & parameter \\
\hline measuring & Current & Range 0-20A \\
power & Range 0-10kw \\
Calculation & Electric energy & $\begin{array}{c}\text { Four-quadrant energy metering, multi-rate } \\
\text { energy accumulation, maximum demand }\end{array}$ \\
& Voltage crest factor, & \\
monitor & current K factor, voltage & \\
& and current,Imbalance & \\
Measure & Itemized metering circuit & $\begin{array}{c}\text { Separately measured external power supply } \\
\text { circuits, special area power supply circuits }\end{array}$ \\
\hline
\end{tabular}

In the above experimental environment, the real-time energy consumption results of the two data transmission methods are statistically analyzed.

\subsection{Experimental results and analysis}

Under the arranged experimental conditions, the continuous period (24h) was randomly selected for testing, and the energy consumption of the existing data transmission method and the design method of this paper was recorded. The experimental results are shown in the following figure:

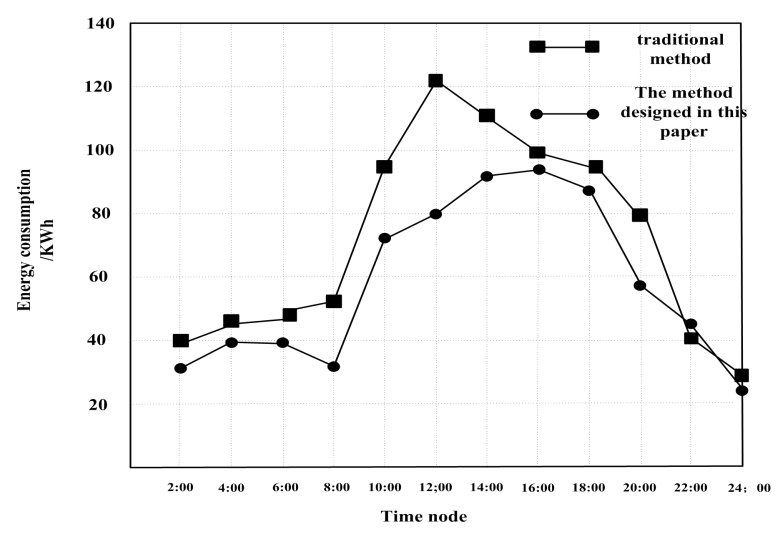


Fig7 Comparison of experimental results

In the selected continuous period, observe and record the energy consumption every $2 \mathrm{~h}$, clear it after recording, and start recording the energy consumption within the next $2 \mathrm{~h}$. During the period of 0: 00-8: 00, during the Internet gap period, the transmission link is basically in a dormant state. Between 8: 00-20: 00, the transmission link begins to continuously transmit marketing analysis data and is at work In this mode, energy consumption is gradually increasing. According to the comparison chart of the experimental results, it can be clearly seen that the current data transmission method consumes more energy than the method in this paper, whether in the sleep state or the working mode. The energy consumption within $24 \mathrm{~h}$ is calculated in detail. The total energy consumption of the existing transmission method is 869 $\mathrm{kWh}$. The total energy consumption of the transmission method in this paper is $693 \mathrm{kWh}$. In 24 hours, the transmission method designed in this paper saves $176 \mathrm{kWh}$ than the existing data transmission methods.

In order to further verify the effectiveness of this system, the traditional system and the new media marketing analysis data transmission accuracy of this system are compared and analyzed, and the comparison results are shown in Figure 8.

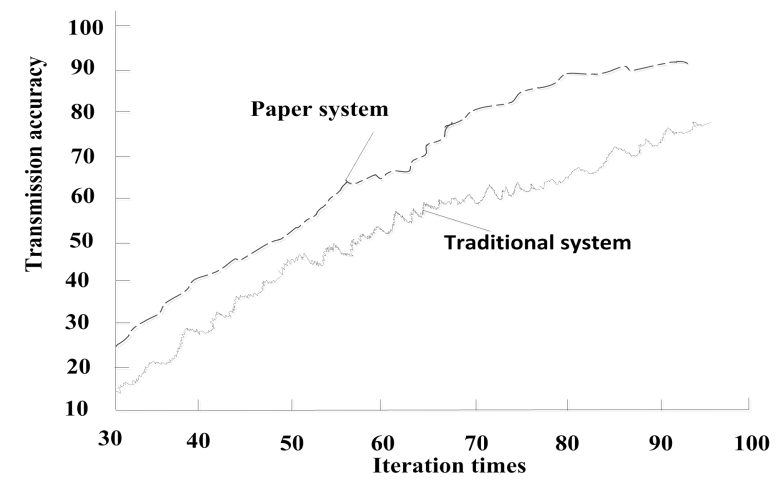

Fig .8 Comparison of transmission accuracy between two systems

According to figure 8, the new media marketing analysis data transmission accuracy of this system is higher than that of the traditional system.

\section{Concluding remarks}

Web4.0 can integrate all digital and information resources that can be integrated to provide users with the best information services under the premise of identification. Data transmission is inseparable during this period. In the context of the whole society advocating energy saving and emission reduction, and building a green business environment, the existing data transmission methods consume too much energy and no longer meet the requirements of a green society. Therefore, a new media marketing analysis based on Web4.0 is designed Data 
transmission method. By designing the structural block diagram of the transmission method, the Steiner tree model for data transmission is established, the transmission channel parameters are designed, and the optimization of the multipath allocation mechanism is completed. The experimental results show that the transmission method designed in 24 hours saves $176 \mathrm{kWh}$ than the existing data transmission methods. This method does not consider the problem of transmission time in the process of verifying the accuracy of data transmission in new media marketing analysis, which results in the increase of data volume and the slower transmission time. Therefore, in the next study, we will further study the data transmission in new media marketing analysis and improve the transmission efficiency.

\section{References}

[1] BAO Shanshan,YANG Chengbing: Optimization of Tourist Destination New Media Marketing Strategies — An Empirical Study of Wuhu City. Journal of Anhui Electrical Engineering Professional Technique College,24(02):61-66(2019).

[2] WANG Su-lan: On Influence of New Media Marketing on Enterprise Performance from the Perspective of Value Co-creation. Journal of Wuhan Commercial Service College ,33(01):50-53(2019).

[3]LI Jiahui: Research on the Current Situation and Strategy of University Library Service Marketing Based on WeChat Public Number-Taking University Libraries in Guangdong Province as an Example. Journal of Jialing University,37(02):110-116(2019).

[4] Zhu Jingwen, Yang Wancheng: Research on Knowledge Network of Publishing Marketing in China :Bibliometric Analysis of CSSCI. Publishing Journal,26(05):69-74(2018).

[5] Xu Jiao,Chen Xiao-jing,Yao Pei-jun: "Tourist attractions + new media" :a new marketing Yangzhou Park. Jiangsu Commercial Forum,(02):26-32(2018).

[6] Li Yan: Research on Marketing Innovation of Marine Products in the Beibu Gulf in the New Media Era. Reformation \& Strategy,34(09):45-48(2018).

[7] WANG Jian-xi,YUE Yuan: Research on Optimization Method of Wireless Communication Data Transmission Energy Consumption in Cloud Environment. Telecom Power Technologies,36(10):37-38(2019).

[8] LI Yanxiang,CAO Xiaofeng: Research on the Reliable Data Transmission Method of Body Area Network Based on Network Coding. Software Engineer,22(08):1-3(2019).

[9] LI Kong-ze,OU Chun-yan: A statistical method-based data transmission experiment system for long-distance communication Electronic Design Engineering,27(14):163-166+171(2019).

[10] LIU Yan,LIU Yun-yu: Time Delay Can Tolerate Data Transmission Efficiency Real-Time Optimization Method Simulation.Computer Simulation,36(11):163-166(2019). 